\section{MUJERES, GÉNERO Y \\ CIENCIAS: ¿UN SEXISMO MODERNO?: TRADUCCIÓN DE “FEMMES, GENRE ET SCIENCES : UN SEXISME MODERNE?" DE NICKY LE FEUVRE}

WOMEN, GENDER AN

SCIENCE: A MODERN

SEXISM?: TRANSLATION

OF "FEMMES, GENRE ET

SCIENCES: UN SEXISME MODERNE?" OF NICKY LE FEUVRE

CLAUDIA ELISA LÓPEZ MIRANDA ${ }^{1}$

Le Feuvre, N. (2013). Femmes, genre et sciences : un sexisme moderne? En: Maruani M. (Ed.), Travail et genre dans le monde. L'etat des savoirs (pp. 4 19-427). Paris: La Découverte.

' Universidad Autónoma del Estado de México, México. Correo electrónico: ely_lopezm@hotmail.com
La investigación sobre desigualdad por sexo en las ciencias alcanza un desarrollo exponencial hacia los años ochenta. Un primer corpus se relaciona con el proceso científico en sí mismo, reflexiona en torno a los efectos de las adscripciones sociales de los individuos en el proceso de producción de conocimiento, así como sobre el rol histórico de las ciencias en la reproducción y la legitimación de las desigualdades de sexo. Un segundo corpus se interesa por los recorridos profesionales de las mujeres y especialmente, por sus dificultades de acceso a los escalones superiores de la carrera científica y las posibles especificidades en su manera de "hacer ciencia". Estas investigaciones son desarrolladas a raíz de los movimientos sociales de los años 70 , contra la exclusión de las mujeres en el ejercicio del poder. Sin embargo, las actividades científicas ocupan un lugar estratégico en las "economías del conocimiento" y es por eso que el análisis del lugar de la mujer en la ciencia 
es particularmente interesante desde

el punto de vista del género.

\section{Un campo de investigación marcado por la pluralidad de perspectivas de análisis}

Un primer corpus cuestiona las pretensiones de objetividad en la producción de conocimientos científicos, que niegan las interferencias vinculadas a características de adscripción social de los investigadores. Esta corriente afirma que, en contraste con la neutralidad axiológica pregonada por la ciencia legítima, los procesos de producción de conocimiento no escapan absolutamente de las influencias de la filiación social, incluido el género (Haraway, 1988 ; Harding, 1987). Las llamadas teorías de punto de vista cuestionan la solidez del conocimiento científico producido por un pequeño grupo social y sugieren que una mayor presencia de mujeres en las profesiones de investigación aumentaría la fiabili- dad y la diversidad del conocimiento (Comisión Europea, 2012b). Este postulado hace énfasis en la forma en que el denominado conocimiento "científico" ha participado históricamente en la naturalización y legitimación de las desigualdades de género (Gardey y Löwy, 2000; Le Doeuff, 1998; Mosconi, 1994).

Sin estar completamente cerrado a las contribuciones de la teoría del punto de vista, un segundo corpus está más interesado en las experiencias de las mujeres en los cursos de formación y en las profesiones científicas. En un contexto donde el número de mujeres calificadas continúa creciendo (ver Baudelot y Establet, capítulo 12 ) y donde el desarrollo del empleo en el sector de investigación (Investigación y Desarrollo) es una prioridad política, el carácter trunco de las carreras académicas de las mujeres es una nueva área de preocupación. Los argumentos movilizados a favor de las mujeres en la ciencia han evolucionado con 
$368 \longrightarrow$ La ventana, Núm. 54/2021

el tiempo: los principios de justicia social (Junter, 2004) han cedido un poco a las preocupaciones sobre la eficiencia económica (Comisión Europea, 2012b).

\section{Las carreras científicas de las mujeres}

Las investigaciones sobre el lugar de las mujeres en las ciencias parten, invariablemente, de una observación empírica (ver la Figura I): la de su "pérdida" a medida que ascienden en la jerarquía socioprofesional. Aunque los gráficos de tijera tienen un efecto visual particularmente poderoso, su interpretación no es fácil. De hecho, antes de concluir que las mujeres son eliminadas de las profesiones científicas, aún es necesario observar algunos datos sobre el atractivo relativo de estas profesiones con respecto a las otras posibilidades de empleo disponibles para los doctores (Studer, 2012). Sin embargo, es probable que este atractivo varíe dependiendo de la disciplina y el contexto social. Como resultado, tales gráficos a menudo se usan de una manera algo abusiva, como si hicieran posible explicar la "evaporación" de las mujeres en un curso relativamente marcado, que comprende etapas sucesivas de calificación, reclutamiento, retención y promoción en la que todas las personas calificadas necesariamente desearían participar. El análisis de este proceso se basa con mayor frecuencia en conceptos desarrollados para dar cuenta de la feminización de otros oficios especializados, como: el "techo de cristal", el "piso adhesivo", el "tubo perforado", etc. cuya utilidad y limitaciones han sido enfatizadas en otros estudios (Buscatto y Marry, 2009). 


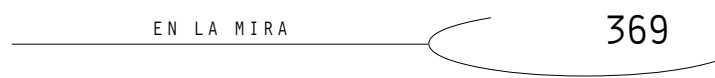

Figura I. Proporción de hombres y mujeres en las diferentes etapas de la trayectoria académica, Unión europea del 27, 2002-2006

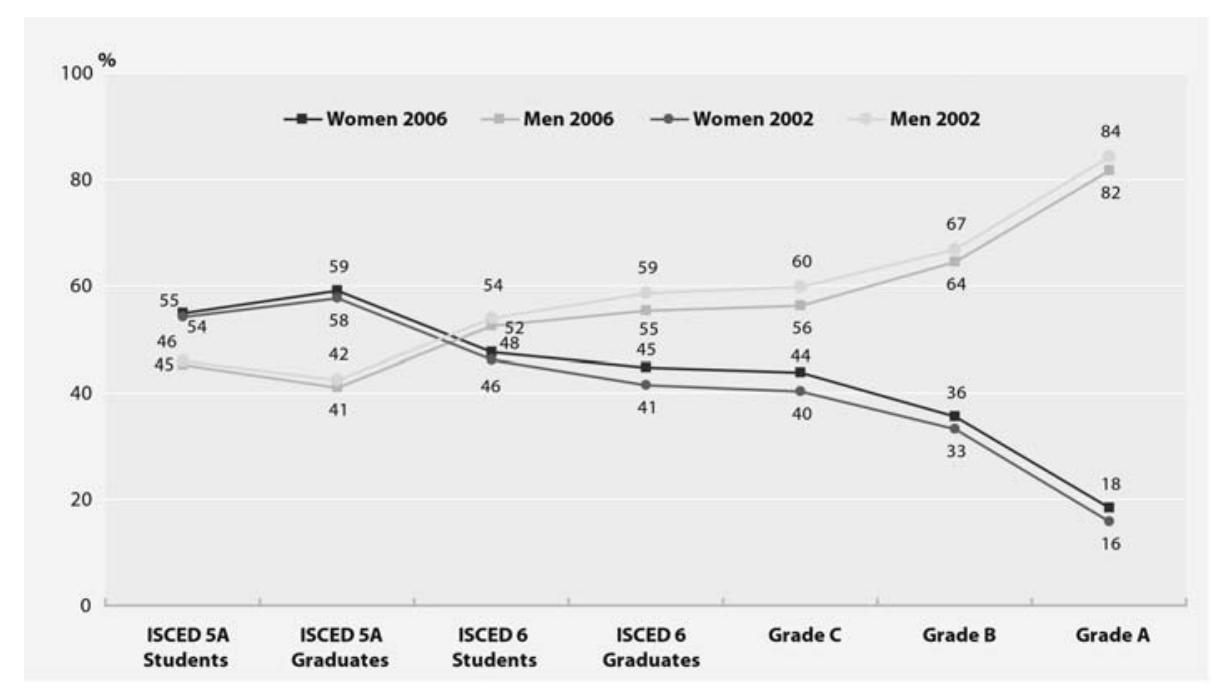

Fuente: Comisión Europea (2009, p. 73).

\section{Glosario}

ISCED 5A Students: Estudiantes nivel maestría.

ISCED 5A Graduates: Titulados de maestría.

ISCED 6 Students: Doctorantes

ISCED 6 Graduates: Titulados de doctorado.

Grade C: Personnel académique debutante (Posdoctorantes)

Grade B: Personal académico de nivel intermedio (Profesores o equivalentes)

Grade A: Personal académico de nivel superior (Profesores o equivalentes) 
$370 \longrightarrow$ La ventana, Núm. 54/2021

\section{El estado de la cuestión}

En 20 I 2, la Comisión Europea publicó un metaanálisis de la investigación realizada sobre el tema "género y ciencia” desde 1980 (Caprile et al., 20 I2). Junto con una base de datos en línea (Comisión Europea, 20 I2a), esta investigación proporciona una fuente extremadamente rica de información sobre la evolución de los problemas de investigación en este campo. Basadas en el principio de que la lentitud del proceso de feminización de las profesiones de investigación está claramente fuera de sintonía con los valores meritocráticos promovidos dentro de las propias instituciones científicas, tres observaciones cruzan todas las publicaciones enumeradas.

Para empezar, las materias científicas presentan un proceso lento, pero universal, de feminización. El aumento del nivel de cualificación de las mujeres se traduce en un crecimiento casi mecánico de su representación en el seno de las instituciones científicas. Sin embargo, el ritmo de esta feminización se mantiene muy por debajo de lo observado en otros sectores de actividad (Caprile et al, 20 I 2). Finalmente, el ritmo de crecimiento de las mujeres en el campo científico varía considerablemente según las disciplinas (Delavault, Boukhobza, Hermann y Conrad, 2002; de Cheveigné, 2009) y según los contextos sociales (Beaufays y Kraïs, 2005; Fassa y Kradolfer, 20I0; Krefting, 2008; Le Feuvre, 2009; Meulders, O’Dorchai y Simeu, 20 I2; Ollagnier et Solar, 2006; Siemienska y Zimmer, 2007).

Este estado de cosas confirma la presencia de una pluralidad en los marcos explicativos desarrollados para dar cuenta de la subrepresentación de las mujeres en el seno del campo científico. Si bien este fenómeno está adquiriendo gradualmente el estatus de un problema social legítimo, no hay consenso sobre qué es exactamente "problemático" (Garforth y Kerr, 2009). Es 
posible identificar al menos tres formas distintas de abordar esta cuestión (Le Feuvre, 20I0), que tienen que ver con la naturaleza de las encuestas realizadas, el tipo de datos empíricos recopilados y las medidas adoptadas para promover las carreras científicas de las mujeres.

Un primer cuerpo de trabajo parte del principio de que son las propias mujeres quienes "plantean un problema”, en el sentido de que luchan por cumplir con los requisitos específicos de las carreras científicas, a veces debido a sus características biológicas (Ecklund, Lincoln y Tansey, 20 I2) pero sobre todo a sus "deficiencias" en la socialización. Un segundo corpus plantea la hipótesis de que el problema radica más bien en los "regímenes de género" a nivel social. Aquí, son las condiciones de vida estructuralmente impuestas a las mujeres, en particular su asignación normativa a las actividades de cuidado, lo que explicaría las dificultades que experimentan al subir la escalera de las profesiones científicas, calificadas como "masculinas" y "elitistas" (Zarca, 2006). Por lo tanto, es la "falta de disponibilidad" de las mujeres lo que constituye el principal objeto de análisis. Una tercera perspectiva desplaza la atención prestada de las personas hacia las instituciones científicas (Backouche, Godechot y Naudier, 2009; Musselin y Pigeyre, 2008), analizando más de cerca las lógicas organizacionales y sus efectos potencialmente discriminatorios sobre las mujeres (y otros grupos minoritarios). Si bien cada una de estas perspectivas proporciona una idea de la relativa exclusión de las mujeres de los niveles superiores de la ciencia, ninguna de ellas es completamente satisfactoria como paradigma explicativo exclusivo.

\section{Aportes y límites de las perspecti- vas de análisis}

Primero, mucha de la investigación sobre la ausencia de mujeres en el piná- 
$372 \longrightarrow$ La Ventana, núm. 54/2021

culo de la jerarquía científica parte de la premisa de que el proceso de socialización de género hace que las mujeres, tendencialmente, estén menos conformes que los hombres con las demandas de la carrera ascendente. Dichos análisis devienen frecuentemente en medidas de tutoría para "corregir" esos "errores" de socialización, para lograr que las mujeres estén tan conformes como se supone que sus homólogos masculinos lo están desde el principio. El principal problema con esta perspectiva es que se basa predominantemente en estereotipos sexistas. De hecho, hasta la fecha, ninguna investigación seria ha permitido validar empíricamente las creencias según las cuales las mujeres graduadas han sido socializadas sistemáticamente de tal manera que los comportamientos asociados a lo científico (curiosidad, inventiva, etc.) sean menos evidentes en ellas que sus homólogos masculinos. Tales enfoques tienden a ignorar la historicidad de los procesos de socialización de género y las condiciones cambiantes de la producción científica. Esta falta de validación empírica de los supuestos básicos es en gran medida válida para los enfoques que enfatizan la "falta de disponibilidad" de las mujeres. Dichos enfoques subrayan correctamente la naturaleza de la actividad científica que consume mucho tiempo y destacan los criterios de excelencia, basados en la productividad y el "autoemprendimiento" (Ehrenberg, 1991), que preside los reclutamientos y promociones en el mundo académico. Pero debido a las responsabilidades familiares que continúan pesando sobre sus hombros, las mujeres tendrían objetivamente más dificultades que los hombres para cumplir con estos criterios de excelencia y, por lo tanto, (y con bastante legitimidad) serían excluidas del proceso de nombramiento y/o promoción.

Dichos análisis generalmente van acompañados de recomendaciones: aportes y límites de las perspectivas de análisis en términos del desarrollo 


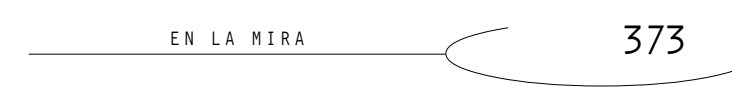

de las carreras profesionales femeninas, a fin de mejorar las posibilidades de "reconciliación" (Fusulier y Rio Carrel, 2012). Aquí aparecen dos limitaciones principales. En primer lugar, muchas otras profesiones calificadas comparten la naturaleza que consume mucho tiempo de las profesiones de investigación, sin experimentar tan baja feminización (Lapeyre, 2006). Segundo, ninguna encuesta empírica puede validar la hipótesis de una influencia negativa de las responsabilidades familiares en el desempeño de las mujeres científicas, ni en el progreso de su carrera. Por ejemplo, en el contexto de América del Norte, ni el estado civil ni el número de hijos se correlacionan con las medidas habituales de productividad científica, incluido el número de publicaciones en revistas revisadas por pares (Yu y Shauman, 1998; 2004) Del mismo modo, en el caso francés, el hecho de ser madre (o no) no parece determinar las posibilidades de ascenso de
Maîtresses de conférences al puesto de Professeure (Latour y Le Feuvre, 2006) este no es el caso en Alemania, por ejemplo (Le Feuvre, 2009). Sin embargo, existe una creencia generalizada sobre la influencia universal de las "discapacidades" de las mujeres casadas/madres en la carrera por el ascenso. Una vez más, las soluciones propuestas para resolver este (falso) problema corren el riesgo de reforzar las creencias y reproducir los estereotipos sobre el pobre "desempeño" de las mujeres científicas.

La tercera perspectiva de análisis fue desarrollada a raíz de una publicación en la prestigiosa revista Nature. En este artículo, los autores muestran que, al solicitar una beca posdoctoral, las mujeres suecas debían tener 2.5 veces más publicaciones que sus homólogos masculinos para tener las mismas posibilidades de obtener fondos (Wenneras y Wold, 1997). En un entorno profesional que se enorgullece de respetar escrupulosamente las 
$374 \longrightarrow$ La ventana, Núm. 54/2021

reglas de la meritocracia y en un país que se considera particularmente favorable a la igualdad de género, iesta investigación ha tenido el efecto de una bomba! Incluso si estos resultados han sido matizados después (Marsh, Bornmann, Daniel, Mutz y O'Mara, 2009), no obstante, han allanado el camino para una serie de investigaciones que cambian la definición de lo que es problemático, desde las propias mujeres hasta el análisis del funcionamiento de las instituciones científicas. Lejos de centrarse en lo que "son" las mujeres (debido a su socialización), o en lo que "hacen" (debido a su sobrecarga doméstica), este trabajo enfatiza sobre todo cómo los líderes académicos evalúan a las mujeres, a través del prisma de género (Le Feuvre, 2010). Este reenfoque en las lógicas institucionales puede ilustrarse con una encuesta de una muestra de profesores (en física, biología y química) en los Estados Unidos (Moss-Racusin, Dovidio, Brescoll, Graham y
Handelsman, 2012). Frente a un Curriculum Vitae idéntico, asignado aleatoriamente a un nombre masculino o femenino, los funcionarios académicos (de ambos sexos) evaluaron sistemáticamente las solicitudes masculinas (supuestamente) de manera más favorable que las atribuidas a las mujeres, tanto desde el punto de vista de las habilidades, como el del salario que se ofrecerá en caso de contratación. Esta tendencia a favorecer a los candidatos masculinos se correlaciona positivamente con puntuaciones más altas en una escala de calificación de "sexismo moderno" (Swim, Aikin Hall y Hunter, 1995). Dicha investigación sugiere que, sea lo que sea que hagan, las mujeres son vistas como menos aptas para actividades científicas que los hombres y esta "diferencia" percibida es lo que en última instancia contribuye a descalificarlas en las etapas críticas de la ciencia. Por lo tanto, parece obvio que las medidas adoptadas para pro- 


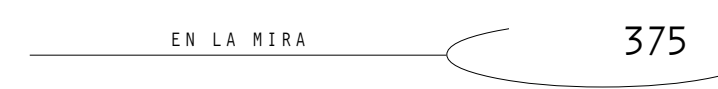

mover a las mujeres en la ciencia deberían centrarse menos en las mujeres mismas y más en los procedimientos institucionales, a fin de eliminar los prejuicios sexistas, que a veces son muy sutiles.

Moss-Racusin, Dovidio, Brescoll, Graham y Handelsman (2012) muestran, por ejemplo, que la tendencia a juzgar a los hombres más "competentes" para un puesto de gerente de laboratorio va acompañada de una tendencia a juzgar las solicitudes atribuidas a las mujeres como más "simpáticas".

Los prejuicios sexistas no se manifiestan necesariamente "a la antigua" por una abierta hostilidad hacia las mujeres, sino más bien por una evaluación negativa y en gran medida inconsciente de sus capacidades para ajustarse a los comportamientos esperados de un investigador de alto nivel y esto independientemente de sus habilidades, disponibilidad o trabajo real y certificado.

Para explicar la exclusión de las mujeres de los niveles superiores de las profesiones científicas, la mayoría de las investigaciones se centran a veces en factores individuales, en influencias sociales o en prácticas institucionales. Sin embargo, una aclaración de los postulados de lo que es "problemático" ayudaría a comprender los mecanismos sociales que funcionan en esta área.

Hay muchas razones para creer que la motivación de las mujeres para invertir en ciencia y su determinación para ascender en la escala de las carreras académicas depende estrechamente de la capacidad de las instituciones académicas para juzgarlas y tratarlas de manera justa. Un análisis más profundo de la compleja relación entre la lógica institucional de excluir a las mujeres de los puestos científicos de responsabilidad y la lógica voluntaria de "autoexclusión" de las mujeres de un entorno profesional que resulta ser sutilmente hostil a su presencia, cons- 
$376 \longrightarrow$ LA VENTANA, NúM. 54/2021

tituye sin lugar a duda, uno de los principales desafíos de la investigación futura en este campo.

\section{Bibliografía}

BACKOUCHE, I., GOdeCHOt, O. y NAUdier, D. (2009). Un plafond à caissons: les femmes à l'EHESS. Sociologie du travail, 5 I (2), 253-274.

Baudelot, C. y establet, R. (20/3). Écoles: égalité et inégalités à l'échelle du monde. En M. Maurani (Dir.), Travail et genre dans le monde. París: La Découverte.

Beaufays, S. y Kraïs, B. (2005). Femmes dans les carrières scientifiques en Allemagne, les mécanismes cachés du pouvoir. Travail, genre et sociétés, (14), 4968.

Buscatto, M. y Marry, C. (2009). Le plafond de verre dans tous ses éclats. La féminisation des professions supérieures au XX e siècle. Sociologie du travail, 5 I (2), 170- 182

Caprile, M., Addis, E., Castaño, C., KlinGe, I., LARIOS, M., Meulders, D. ET AL. (EDS.) (20I2). Meta-analysis of Gender and Science Research. Synthesis Report. Brucelas: Commission européenne, DG Recherche \& Innovation.

De CheVeigné De, S. (2009). The career paths of women (and men) in French research. Social Studies of Science, (39), I13-136.

Commission Européenne. (2009). She Figures 2009. Statistics and Indicators on Gender Equality in Science. Brucelas: DG Recherche $\varepsilon$ Innovation.

Commission Européenne. (20 I 2a). Base de données.

www.genderandscience.org. Commission Européenne. (20I2b). Structural Change in Research Institutions. Enhancing Excellence, Gender Equality and Efficiency in Research and Innovation. 


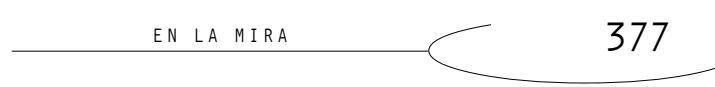

Brucelas: DG Recherche $\varepsilon$ Innovation.

Delavault, H., Boukhobza, N., Hermann, C. y Conrad C. (2002), Les Enseignantes-chercheuses à l'Université. Demain la Parité? París: L'Harmattan.

ECKlund, H. E., Lincoln, A. E. y Tansey, C. (20I2). Gender segregation in elite academic science. Gender $\mathcal{E}$ Society, 26(5), 693-7I 7.

EhrenberG, A. (1991). Le Culte de la performance. París: CalmannLévy.

Fassa, F. y KRADOLFer, S. (Dir.) (20I0). Le Plafond de fer à l'université. Femmes et carrières. Ginebra: Seismo.

Fusuler, B. y Rio Carrel Del, M. (20 I2). Chercheur-e-s sous haute tension! Vitalité, compétitivité, précarité et (in)compatibilité travail/famille. Louvain-la-Neuve: Presseus universitaires de Louvain.

Gardey, D. y Löwy, I. (Dir.) (2000).
L'Invention du naturel. Les sciences et la fabrication du féminin et du masculine. París: Éditions des archives contemporaines.

Garforth, L. y Ker, R A. (2009). Women and science. What's the problem? Social Politics, I6(3), 380-403.

Harding, S. (1987). The Science Question in Feminism. Cornell: Cornell University Press.

Haraway, D. (1988). Situated knowledges. The science question in feminism and the privilege of partial perspective. Feminist Studies, 14(3), 575-599. Junter, A. (2004). L'égalité professionnelle entre les femmes et les hommes: une exigence politique au coeur du droit des femmes. Travail, genre et sociétés, (12), I9|-202.

Krefting, L. A. (2008). Intertwined discourses of merit and gender. Evidence from academic employment in the USA. Gender, 
$378 \longrightarrow$ La Ventana, núm. $54 / 2021$

Work and Organizations, 10(2),

260-278.

Lapeyre, N. (2006). Les Professions face

aux enjeux de la féminisation.

Toulouse: Octarès.

Latour, E. y Le Feuvre, N. (2006). Les

carrières universitaires françaises

à l'épreuve du genre, En E.

Ollaginer y C. Solar (Dir.), Parcours

de femmes à l'Université.

Perspectives internationals ( $\mathrm{pp}$.

159-188). París: L'Harmattan.

Le Doeuff, M. (1998). Le Sexe du savoir.

París: Aubier.

Le Feuvre, N. (2009). Exploring

women's academic careers in

cross-national perspective.

Lessons for equal opportunity

policies. Equal Opportunities

International, 28(I), 9-23.

Le Feuvre, N. (2010). Les carrières universitaires à l'épreuve du genre: éléments de conclusion. En F. Fassa y S. Kradolfer (Dir.), Le plafond de fer à l'université.
Femmes et carrières (pp. 225242). Ginebra: Seismo.

Marsh H, W., Bornmann, L., Daniel, H. D., Mutz, R. y O'MARA, A. (2009).

Gender effects in peer reviews of grant proposals. A comprehensive meta-analysis comparing traditional and multilevel approaches. Review of Educational Research, 79(3), 1290-1326.

Meulders, D., O'dorchal, S. y Simeu, N. (20I2). Alma Mater, Homo sapiens II - Les inégalités entre femmes et hommes dans les universités francophones de Belgique. Brucelas: Éditions du Dulbea.

Mosconi, N. (1994). Femmes et rapports au savoir. La société, l'école et la división sexuelle des savoirs. París: L'Harmattan.

Moss-Racusin, C. A., Dovidio, J. F., Brescoll, V. L., Graham, M. J. y Handelsman, J. (20I2). Science faculty's subtle gender biases fa- 


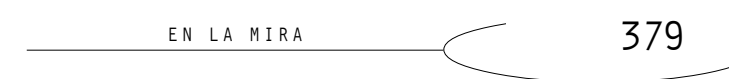

vor male students. Proceedings of the national academy of sciences of the USA. Www.pnas.org/ content/early/20/2/09//4/ 1211286109.

Musselin, C. y Pigeyre F. (2008). Les effets du recrutement collégial sur la discrimination: le cas des recrutements universitaires. Sociologie du travail, (50), 48-70. Ollagnier, E. y Solar, C. (Dir.). (2006). parcours de femmes à l'Université. Perspectives internationals. París: L'Harmattan.

SiemienskA, R. y Zimmer, A. (Dir.) (2007). Gendered Career Trajectories in Academia in Cross-national Perspective. Varsovia: Scholar. Studer, M. (2012). Étude des inégalités de genre en début de carrière académique à l'aide de méthodes innovatrices d'analyse de données séquentielles (Tesis de doctorado). Université de Genève, Ginebra.
Swim, J. K., AlkIN, K. J., Hall, W. S. y Hunter, B. A. (1 995). Sexism and Racism. Old-fashioned and modern prejudices. Journal of Personality and Social Psychology, (68), 199-214.

Wenneras, C. y Wold A. (1997). Nepotism and sexism in peer review. Nature, (387), 34 I-343.

Yu, X. y Shauman, K. (1 998). Sex differences in research productivity: new evidence about an old puzzle. American Sociological Review, (63), 770847.

Yu, X. y Shauman, K. (2004). Women in Science. Career, Process and Outcomes. Cambridge: Harvard University Press.

ZARCA, B. (2006). Mathématicien: Une profession élitaire et masculine. Sociétés contemporaines, (64), 4165. 